\title{
124. Über die Definition der Shodaschen Diskriminante eines normalen einfachen hyperkomplexen Systems.
}

\author{
Von Tadasi Nakamura. \\ Mathematisches Institut, Kaiserliche Universität zu Tokyo. \\ (Comm. by T. TaKagi, M.I.A., Oct. 12, 1934.)
}

In einer Arbeit ${ }^{1)}$ hat $\mathrm{K}$. Shoda Diskriminante eines normalen einfachen hyperkomplexen Systems definiert. Dabei hat er aber die Existenz einer Minimalbasis einer Maximalordnung in bezug auf die Maximalordnung des Zentrums vorausgesetzt. Im allgemeinen musste er sie im Kleinen definieren. ${ }^{2}$ ) In dieser Arbeit gebe ich eine direkte Definition im Grossen an.)

Es sei $\mathfrak{S}$ eine normale einfache Algebra über einem algebraischen Zahlkörper $K$ endlichen Grades, $K_{0}$ ein Unterkörper von $K$ derart, dass eine Maximalordnung $\mathfrak{D}$ von $\mathfrak{S}$ eine Minimalbasis $\left(E_{1}, E_{2}, \ldots \ldots, E_{N}\right)$ in bezug auf die Maximalordnung von $K_{0}$ bezitzt. Die Existenz eines solchen Unterkörpers ist klar, da der rationale Zahlkörper die Eigenschaft von $K_{0}$ hat. Es sei ferner $\left(e_{1}, e_{2}, \ldots, e_{n}\right)$ eine beliebige Basis von $\mathcal{S}$ in Bezug auf das Zentrum $K$. Dann gibt es Matrizen $A_{i}, B_{j}$ in $K$, die den Gleichungen

$$
E_{i}(e)=(e)\left(\begin{array}{cc}
a_{i i}^{1} \ldots & a_{i n}^{1} \\
\vdots & \vdots \\
a_{i 1}^{n} \ldots & a_{i n}^{n}
\end{array}\right)=(e) A_{i}, \quad(e) E_{j}=(e)\left(\begin{array}{c}
b_{1 j}^{1} \ldots b_{n j}^{1} \\
\vdots \\
b_{1 j}^{n} \ldots . . b_{n j}^{n}
\end{array}\right)=(e) B_{j}
$$

genügen, wo $(e)$ die einzeilige Matrix $\left(e_{1}, e_{2}, \ldots, e_{n}\right)$ bedeutet.

Man bilde nun die Matrix

$$
M\left[\begin{array}{l}
E_{1} \ldots E_{N} \\
e_{1} \ldots e_{n}
\end{array}\right]=\left(\sum_{k} a_{i p}^{k} b_{k j}^{q}\right),
$$

wo $(p, q)$ bzw. $(i, j)$ Zeilen- bzw. Spaltenindex ist. Dann kann man beweisen :

Der Rang von $M\left[\begin{array}{l}E_{1} \ldots E_{N} \\ e_{1} \ldots e_{n}\end{array}\right]$ ist gleich $n^{2}$. Der grösste gemeinsame Teiler aller Unterdeterminanten $n^{2}$-ten Grades von $M\left[\begin{array}{l}E_{1} \ldots E_{N} \\ e_{1} \ldots e_{n}\end{array}\right]$ ist die Shodasche Diskriminante von $\mathfrak{S}$.

1) K. Shoda: Diskriminantensatz für normale einfache hyperkomplexe Systeme, Proc. 10 (1934), 315. Diskriminantenformel für normale einfache hyperkomplexe Systeme, Proc. 10 (1934), 318.

2) Vgl. Anmerkung 1) in der vorstehenden Arbeit von $\mathrm{K}$. Shoda und mir.

3) Die Anregung zu dieser Arbeit verdanke ich K. Shoda. 
Zum Beweis betrachten wir $N^{2}$ Elemente $E_{i} x E_{j}$ mit dem Unbestimmten $x=\sum_{p=1}^{n} e_{p} x_{p}$, unter denen $n^{2}$ Elemente linear unabhängig bezüglich $K$ sind.") Da aber

$$
\left(E_{1} x E_{1}, \ldots E_{i} x E_{j}, \ldots, E_{N} x E_{N}\right)=\left(e_{1} x_{1}, \ldots e_{q} x_{p}, \ldots, e_{n} x_{n}\right) M\left[\begin{array}{l}
E_{1} \ldots E_{N} \\
e_{1} \ldots e_{n}
\end{array}\right],
$$
ist, so ist der Rang von $M\left[\begin{array}{l}E_{1} \ldots E_{N} \\ e_{1} \ldots e_{n}\end{array}\right]$ gleich $n^{2}$.

Wir beweisen nun, dass der grösste gemeinsame Teiler $\mathfrak{D}\left[\begin{array}{l}E_{1} \ldots E_{N} \\ e_{1} \ldots e_{n}\end{array}\right]$ aller Unterdeterminanten $n^{2}$-ten Grades von $M\left[\begin{array}{l}E_{1} \ldots E_{N} \\ e_{1} \ldots e_{n}\end{array}\right]$ von der Wahl der Basis $\left(e_{1}, e_{2}, \ldots, e_{n}\right)$ unabhängig ist. Nimmt man nämlich eine andere Basis $\left(e_{1}^{\prime}, e_{2}^{\prime}, \ldots, e_{n}^{\prime}\right)$ an, wo $(e)=\left(e^{\prime}\right) P$ ist, so folgen aus

$$
E_{i}\left(e^{\prime}\right)=\left(e^{\prime}\right) A_{i}^{\prime}, \quad\left(e^{\prime}\right) E_{j}=\left(e^{\prime}\right) B_{j}^{\prime}
$$

ersichtlich die Gleichungen

$$
A_{i}^{\prime}=P A_{i} P^{-1}, \quad B_{j}^{\prime}=P B_{j} P^{-1}, \quad B_{j} A_{i}=P B_{j} A_{i} P^{-1} .
$$

Da der Koeffizient von $B_{j} A_{i}$ an der Stelle $(q, p)$ gleich $\sum_{k} a_{i p}^{k} b_{k j}^{q}$ ist, so ist, wie man leicht ausrechnen kann,

$$
M\left[\begin{array}{l}
E_{1} \ldots E_{N} \\
e_{1}^{\prime} \ldots e_{n}^{\prime}
\end{array}\right]=\left(P^{-1} \times P\right) \cdot M\left[\begin{array}{l}
E_{1} \ldots E_{N} \\
e_{1} \ldots e_{n}
\end{array}\right],
$$

wo $P^{-1} \times P$ das Kroneckersche Produkt von $P^{-1}$ und $P$ bedeutet. Da aber $\left|P^{-1} \times P\right|=1$ ist, so folgt hieraus

$$
\mathfrak{D}\left[\begin{array}{l}
E_{1} \ldots E_{N} \\
e_{1} \ldots e_{n}
\end{array}\right]=\mathfrak{D}\left[\begin{array}{l}
E_{1} \ldots E_{N}^{\prime} \\
e_{1}^{\prime} \ldots e_{n}^{\prime}
\end{array}\right] \text {. }
$$

Wir beweisen nun, dass $\mathfrak{T}\left[\begin{array}{l}E_{1} \ldots E_{N} \\ e_{1} \ldots e_{n}\end{array}\right]$ von der Wahl der Minimalbasis $\left(E_{1}, E_{2}, \ldots, E_{N}\right)$ unabhängig ist. Ist nämlich $\left(E_{\mathrm{I}}^{\prime}, E_{2}^{\prime}, \ldots, E_{N}^{\prime}\right)$ eine andere Minimalbasis und

$$
\left(E_{1}^{\prime}, E_{2}^{\prime}, \ldots, E_{N}^{\prime}\right)=\left(E_{1}, E_{2}, \ldots, E_{N}\right) Q,
$$

so sind $Q$ und $Q^{-1}$ beide ganzzahlig. Dann ergibt sich ferner leicht

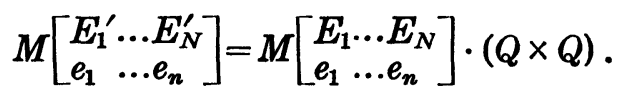

Da $Q \times Q$ ganzzahlig ist, so ist jede Unterdeterminante $n^{2}$-ten Grades von $M\left[\begin{array}{lll}E_{1}^{\prime} & \ldots & E_{N}^{\prime} \\ e_{1} & \ldots & e_{n}\end{array}\right]$ eine lineare Kombination der Unterdeterminanten

4) K. Shoda : Ein Kriterium für normale einfache hyperkomplexe Systeme, Proc. 10 (1934), 195. 
$n^{2}$-ten Grades von $M\left[\begin{array}{l}E_{1} \ldots E_{N} \\ e_{1} \ldots e_{n}\end{array}\right]$, deren Koeffizienten ganz sind. Also

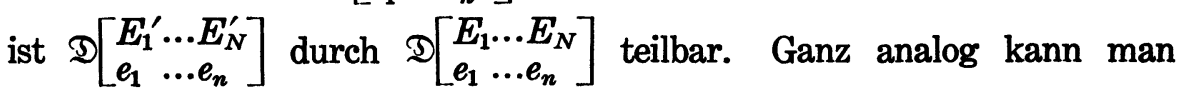

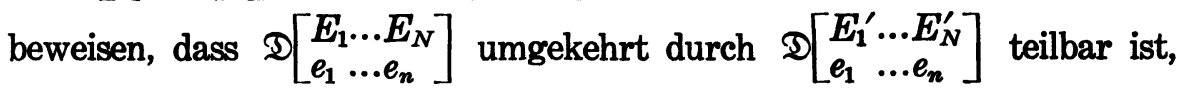
da $Q^{-1}$ ganzzahlig ist. Daher ist

$$
\mathfrak{D}\left[\begin{array}{lll}
E_{1} & \ldots & E_{N} \\
e_{1} & \ldots & e_{n}
\end{array}\right]=\mathfrak{D}\left[\begin{array}{lll}
E_{1}^{\prime} \ldots E_{N}^{\prime} \\
e_{1} & \ldots & e_{n}
\end{array}\right] \text {. }
$$

Wir beweisen endlich, dass das hier definierte, von der Wahl von $\left(E_{1}, E_{2}, \ldots, E_{N}\right), \quad\left(e_{1}, e_{2}, \ldots, e_{n}\right)$ unabhängige Ideal $\mathfrak{D}$ gleich der im Kleinen definierten Shodaschen Diskriminante ist.5) Dafür genügt es zu zeigen, dass der $\mathfrak{p}$-Komponent von $\mathfrak{D}$ gleich der Shodaschen Diskriminante von $\mathfrak{S}_{\mathfrak{p}}$ ist, wo $\mathfrak{p}$ ein beliebiges Primideal in $K$ bedeutet.

Im allgemeinen bilden die $E_{1}, E_{2}, \ldots, E_{N}$ nicht mehr eine Minimalbasis von $\mathfrak{S}_{\mathfrak{p}}$ bezüglich $\left(K_{0}\right)_{\mathfrak{p}_{0}}$, wo $\mathfrak{p}_{0}$ das Primidealvielfache von $\mathfrak{p}$ in $K_{0}$ ist.6) Denn der Rang $\left(\mathfrak{S}_{\mathfrak{p}}:\left(K_{0}\right)_{p_{0}}\right)$ ist im allgemeinen kleiner als $N$. Jedes Element aus op lässt sich als eine lineare Kombination von $E_{1}$, $E_{2}, \ldots, E_{N}$ mit ganzzahligen Koeffizienten aus $K_{\mathfrak{p}}$ darstellen. Ist $\left(w_{1}, w_{2}, \ldots, w_{n}\right)$ eine Minimalbasis von $\mathfrak{o}_{\mathfrak{p}}$ bezüglich $K_{\mathfrak{p}}$, so gibt es ganzzahlige Matrizen $Q, R$ in $K_{\mathfrak{p}}$, die den Gleichungen

$$
\left(w_{1}, w_{2}, \ldots, w_{n}\right)=\left(E_{1}, E_{2}, \ldots, E_{N}\right) Q, \quad\left(E_{1}, E_{2}, \ldots, E_{N}\right)=\left(w_{1}, w_{2}, \ldots, w_{n}\right) R
$$

genügen. ${ }^{7)}$ Jetzt kann man wie oben vorgehen und beweisen, dass der $\mathfrak{p}$-Komponent von $\mathfrak{D}\left[\begin{array}{l}E_{1} \ldots E_{N} \\ e_{1} \ldots e_{n}\end{array}\right]$ gleich $\mathfrak{D}\left[\begin{array}{l}w_{1} \ldots w_{n} \\ e_{1} \ldots e_{n}\end{array}\right]$ ist. Es ist aber

$$
\mathfrak{T}\left[\begin{array}{l}
w_{1} \ldots w_{n} \\
e_{1} \ldots e_{n}
\end{array}\right]=\mathfrak{P}\left[\begin{array}{l}
w_{1} \ldots w_{n} \\
w_{1} \ldots w_{n}
\end{array}\right]
$$

und $\mathfrak{D}\left[\begin{array}{l}w_{1} \ldots w_{n} \\ w_{1} \ldots w_{n}\end{array}\right]$ ist nach der Definition nichts anderes als die Shodasche Diskriminante von $\mathfrak{S}_{p}$.

5) Dass $\mathfrak{D}$ auch von $K_{0}$ unabhängig ist, folgt aus dem zu beweisenden Satz, dass D die Shodasche Diskriminante ist. Einen direkten Beweis kann man auch leicht ausführen.

6) Dabei betrachten wir $\left(K_{0}\right)_{\mathfrak{p}_{0}}$ als Unterkörper von $K_{\mathfrak{p}}$.

7) $Q$ ist im allgemeinen nicht eindeutig bestimmt. 